\title{
The Pricing of Two Newly Invented Swaps in a Jump-Diffusion Model $^{*}$
}

\author{
Zhaojun Yang \\ Department of Financial Engineering, Hunan University, Changsha, 410079, China \\ E-mail: zjyang@hnu.edu.cn \\ and \\ Chunhong Zhang \\ Department of Financial Engineering, Hunan University, Changsha, 410079, China \\ E-mail: chhozhang@gmail.com
}

\begin{abstract}
This paper considers the pricing of contingent claims involved in two new swaps invented by Chinese entrepreneurs, the equity-for-guarantee swap (EGS) and the option-for-guarantee swap (OGS), when the cash flow of a firm that enters into the swaps follows a jump-diffusion process with jump sizes having a double exponential distribution. Using an equilibrium pricing approach, we provide explicit prices of all contingent claims and guarantee costs, where a Nash equilibrium of the game between the insurer and the borrower is derived. We present numerical analysis and find that OGS leads to an earlier default than EGS. As far as the borrower is concerned, EGS is better than OGS while keeping other parties the same. The advantage increases dramatically with the cash flow risk.
\end{abstract}

Key Words: Option-for-guarantee swap; Equity-for-guarantee swap; Guarantee costs; Nash equilibrium.

JEL Classification Numbers: G12, G23.

\section{INTRODUCTION}

On account of financing difficulties faced by small- and medium- sized enterprises (SMEs), Shenzhen High-Tech Investment Guarantee Corporation (SHTIGC) in China invented two new type of swaps, called equityfor-guarantee swap (EGS) and option-for-guarantee swap (OGS). Now, the

* The research for this paper was supported by National Natural Science Foundation of China (Project Nos. 71171078, 71371068 and 71221001).

371

$1529-7373 / 2015$

All rights of reproduction in any form reserved. 
two swaps are getting more and more popular in China. Although the two swaps have appeared in China for a long time, as far as we know, there is no paper in the literature that provides quantitative research on them except Yang and Zhang (2013), Yang and Zhang (2015) and Wang, Yang and Zhang (2015), which consider only EGS and assume the cash flow of the firm that enters into the swap follows a pure diffusion process.

However, as a matter of fact, SMEs and in particular high-tech companies usually undergo a sudden increase or an unexpectedly dramatical decline. For this reason, in this paper we assume the cash flow generated by an SME follows a jump-diffusion model instead of a pure diffusion process. Motivated by Kou (2002) and Fidrmuc, Ciaian and Pokrivcak (2013), we suppose the cash flow is described by a double exponential jump diffusion process. Meanwhile, on account of that OGS is more popular than EGS in China, unlike Yang and Zhang (2013), we consider OGS as well as EGS. Utilizing an equilibrium pricing method, we provide explicit solutions to the pricing of corporate securities under both EGS and OGS, optimal option exercising boundary and the guarantee costs, which are the numbers of the equity (option) allocated by the firm that enters into the EGS (OGS) to an insurer in exchange for the guarantee.

As argued in Kou (2002), two puzzles emerge from many empirical investigations: the leptokurtic feature that the return distributions of assets may have a higher peak and two (asymmetric) heavier tails than those of the normal distribution, and an empirical abnormality called "volatility smile" in option pricing. To incorporate both of them, Kou (2002) proposes, for the purpose of option pricing, a double exponential jump diffusion model, which consists of a continuous part driven by a Brownian motion and a jump part with jump sizes following a double exponential distribution.

There are a lot of papers in the literature studying on the double exponential jump-diffusion model because it usually leads to an explicit conclusion and it seems realistic as well under many situations. For example, Kou and Wang (2003) consider the first passage time to flat boundaries for a double exponential jump diffusion process. Explicit solutions of the Laplace transforms, of both the distribution of the first passage and the joint distribution of the process and its running maxima, are obtained. Sepp (2004) derives explicit formulas for pricing double (single) barriers and touch options with time-dependent rebates assuming that the asset price follows a double-exponential jump diffusion process. Dao (2003) extends the framework of Leland (1994) by examining corporate debt, equity and firm values with jump diffusion processes. Atsuo and Hatsushoge (2009) consider an optimal stopping problem for double exponential jump diffusion processes and derive the value function of the option to postpone and its optimal boundary. Kou and Wang (2004) address option pricing under a double exponential jump diffusion model. 
On account of the above-mentioned reason, it is natural to attack the pricing of contingent claims involved in EGS and OGS under a double exponential jump diffusion model. In particular, there is no formal research on OGS except Yang and Zhang (2015), although OGS is more popular than EGS in practice. Our work reveals that the study on OGS is much more challengeable than that on EGS. For example, as far as OGS is concerned, a game between an insurer and a firm that enters into OGS must be considered.

The remainder of the paper proceeds as follows. Section 2 sets up the model. In section 3 , we explicitly obtain the equilibrium prices of all contingent claims involved in the two swaps and the guarantee costs. We present numerical results in section 4 . Section 5 concludes.

\section{MODEL SETUP}

Consider an SME that has invested in a project, of which the earnings before interest and tax (EBIT), denoted by $\delta$ is observable and independent of the capital structure of the firm. We assume the cash flow is governed by the following jump-diffusion process:

$$
d \delta_{t}=\mu_{\delta} d t+\rho \sigma d Z_{t}^{1}+\sqrt{1-\rho^{2}} \sigma d Z_{t}^{2}+d\left(\sum_{i=1}^{N_{t}} Z_{i}\right), \delta_{0} \text { given }, t \in[0, \infty)
$$

where $\mu_{\delta}$ is the mean growth rate, $\sigma$ is the volatility rate, $Z \equiv\left(Z^{1}, Z^{2}\right)$ is a 2-dimensional standard Brownian motion, $N$ is a Poisson process with the intensity $\lambda$ that is independent of $Z$, both $Z$ and $N$ are defined on a complete filtered probability space $\left(\Omega, \mathcal{F},\left(\mathcal{F}_{t}\right)_{t \geq 0}, \mathbb{P}\right)$ with $\mathcal{F}_{t} \equiv$ $\sigma\left\{Z_{s}^{1}, Z_{s}^{2}, N_{s}, \sum_{i=1}^{N_{s}} Z_{i} ; 0 \leq s \leq t\right\}$ describing the flow of information available to an investor at time $t$, and $Z_{i}, i=1,2, \cdots$, denote independent and identically distributed (i.i.d.) random variables following a double exponential distribution, of which the density function is given by

$$
h(z)=p \cdot \eta_{1} e^{-\eta_{1} z} \mathbf{1}_{\{z \geq 0\}}+q \cdot \eta_{2} e^{\eta_{2} z} \mathbf{1}_{\{z<0\}},
$$

where $p, q \geq 0$ representing the probabilities of upward and downward jumps, are constants, $p+q=1$, and $\eta_{1}, \eta_{2}>0$. Note that the means of the two exponential distributions are $\frac{1}{\eta_{1}}$ and $\frac{1}{\eta_{2}}$ respectively, we therefore have $\xi \equiv \mathbb{E}\left(Z_{i}\right)=\frac{p}{\eta_{1}}-\frac{q}{\eta_{2}}$.

In addition, investors have standard liquid financial opportunities which involves a risk-free asset and a risky market portfolio. The risk-free interest rate is $r>0$. Denote by $M$ the value process of the market portfolio, which 
is governed by the following equation:

$$
d M_{t} / M_{t}=\mu_{m} d t+\sigma_{m} d Z_{t}^{1}, \quad M_{0} \text { given, }
$$

where $\mu_{m}$ and $\sigma_{m}$ are the expected return and the volatility rate of the market respectively. Clearly, the parameter $\rho$ in (1) represents the correlation coefficient between the firm's cash flow and the return of the market portfolio. The value $\rho \sigma$ represents the systematic volatility of the firm's cash flow.

We take it that the original owners of an SME have chosen a capital structure consisting of pure equity and debt in the form of a single console bond, promising a constant coupon payment $b$ to the lender as long as the firm remains solvent. However, unlike the common assumption in the literature, based on many real world situations, we assume that an SME, in contrast to a large company, can not issue any bond directly because of its large default risk.

To solve the debt financing problem, the SME turns to an insurer and signs a three party agreement with a bank and insurer. In the agreement, a bank lends money at a given interest rate to an SME and gets constant coupon payment $b$ from the SME. Once the SME defaults, the insurer gets the salvage value of the assets of the SME and pays all the outstanding interest and principal to the bank instead of the SME. Under OGS, in return for the guarantee, the SME must give the insurer a perpetual American call option to buy a given fraction, denoted by $\varphi^{\circ}$, of equity at a given exercise price $K$ per share ${ }^{1}$ at any time. We call the fraction $\varphi^{o}$ as guarantee cost. This agreement is actually a swap in finance, which is similar with EGS defined in Yang and Zhang (2013). The distinction is that OGS gives an insurer a perpetual American call option on the value of equity instead of a fraction, denoted by $\varphi^{e}$, of equity directly at the very beginning under EGS.

Clearly, the fundamental issue is to determine how much the guarantee $\operatorname{cost} \varphi^{o}$ under OGS or $\varphi^{e}$ under EGS should be to make sure that the agreements are fair. For this aim, in the next section we first consider the pricing of corporate securities under the two swaps and then the two different guarantee costs are derived. Following Goldstein et al. (2001), we assume a simple tax structure that includes personal and corporate taxes, where interest payments are taxed at a personal rate $\tau_{i}$, effective dividends are taxed at $\tau_{d}$, and corporate profits are taxed at $\tau_{c}$, with full loss offset provisions.

\footnotetext{
${ }^{1}$ Without loss of generality, we assume the number of shares of all equity of the SME is just one.
} 


\section{EQUILIBRIUM PRICING OF CORPORATE SECURITIES AND GUARANTEE COSTS}

In this section, we first review the equilibrium pricing theory on the jump-diffusion risk model and then based on the theory, we explicitly obtain the equilibrium prices of all the contingent claims and the guarantee costs defined in previous section.

\subsection{Equilibrium pricing theory under a jump-diffusion model}

In finance, most asset prices are derived from a linear pricing schedule. To determine a linear pricing rule, we must specify a stochastic discount factor. However, the market we consider here is incomplete, i.e. there are infinite stochastic discount factors. To fix one, we can solve a single-agent optimization problem and take the marginal utility of the agent as a special stochastic discount factor. If the agent selected is a representative agent, then we recover the equilibrium stochastic discount factor used in Ingersoll (2006), Goetzmann, Ingersoll and Ross (2003), Yang and Zhang (2013) and essentially also in Merton (1976).

Specifically, we assume the representative agent invests in the standard liquid financial market defined in Section 2 with some given initial wealth $w_{0}$. Therefore, the dynamics of the agent's wealth $W$ is given by

$$
d W_{t}=r W_{t} d t+\theta_{t}\left(d M_{t}-r M_{t} d t\right)-C_{t} d t, \quad t \geq 0,
$$

where $\theta_{t}$ is the number of shares of the risky asset (market portfolio) and $C_{t}$ represents her/his consumption rate at time $t$. The strategy $\left(\theta_{t}, C_{t}\right)_{t \geq 0}$ is said to be admissible for initial wealth $w_{0}$ if the wealth process $W$ given by (3) remains non-negative at all times. The set of all admissible strategies is denoted by $\mathcal{A}\left(w_{0}\right)$. The agent aims to maximize her/his expected total discounted utility of consumption over an infinite planning horizon:

$$
J\left(w_{0}\right)=\sup _{(\theta, C) \in \mathcal{A}\left(w_{0}\right)} \mathbb{E}\left[\int_{0}^{\infty} U\left(t, C_{t}\right)\right]
$$

Usually, to get some reasonably explicit solution, we shall have to assume a simple form of the utility $U$, such as the following constant-relative-riskaversion (CRRA) utility:

$$
U\left(t, C_{t}\right)=\exp (-\Lambda t) \frac{C_{t}^{1-\gamma}}{1-\gamma}
$$

where $\Lambda, \gamma>0$ and $\gamma \neq 1$. Under this assumption, the agent faces an infinite-horizon Merton problem and it is well known that the optimal 
strategy is given by

$$
\theta_{t}^{*}=\frac{\mu_{m}-r}{\sigma^{2} \gamma} \frac{W_{t}}{M_{t}}
$$

and

$$
C_{t}^{*}=\kappa W_{t}, \quad \kappa \equiv \gamma^{-1}\left[\Lambda+(\gamma-1)\left(r+0.5 \eta^{2} / \gamma\right)\right],
$$

where $\eta \equiv\left(\mu_{m}-r\right) / \sigma_{m}$ is the market Sharpe ratio. Substituting the optimal strategy into (3) leads to the following dynamics of the optimal wealth:

$$
W_{t}^{*}=w_{0} \exp \left(\gamma^{-1} \eta Z_{t}^{1}+\left[r-\kappa+0.5 \gamma^{-2} \eta^{2}(2 \gamma-1)\right] t\right) .
$$

After that, we immediately obtain the stochastic discount factor according to Rogers (2013) and Ingersoll (2006) among others:

$$
\pi_{t} \equiv U_{C_{t}}^{\prime}\left(t, C_{t}^{*}\right)=\left(\kappa w_{0}\right)^{-\gamma} \exp \left(-\eta Z_{t}^{1}-0.5 \eta^{2} t-r t\right) .
$$

Consequently, thanks to the proposition in Section 6F of Duffie (2001), the stochastic discount factor $\pi$ corresponds to an equivalent martingale measure $\mathbb{Q}$, for which the density process $\psi$ is given by

$$
\psi_{t}=\exp (r t) \pi_{t} / \pi_{0}=\exp \left(-\eta Z_{t}^{1}-0.5 \eta^{2} t\right) .
$$

Noting that $d \psi_{t}=-\eta \psi_{t} d Z_{t}^{1}$ and utilizing the Girsanov-Meyer theorem, ${ }^{2}$ we can rewrite the cash flow process $\delta$ in (1) as follows:

$$
d \delta_{t}=\mu d t+\rho \sigma d Z_{t}^{\mathbb{Q}}+\sqrt{1-\rho^{2}} \sigma d Z_{t}^{2}+d\left(\sum_{i=1}^{N(t)} Z_{i}\right),
$$

where $\mu \equiv \mu_{\delta}-\rho \sigma \eta$ and $\left(Z^{\mathbb{Q}}, Z^{2}\right)$ is a 2-dimensional standard Brownian motion under the measure $\mathbb{Q}$ satisfying $d Z_{t}^{\mathbb{Q}}=d Z_{t}^{1}+\eta d t$.

It is shown above that the equilibrium pricing is unrelated with the riskaverse index $\gamma$ of the representative agent. We also select an exponent utility (CARA) instead of the power utility (CRRA) discussed above and we obtain the same measure $\mathbb{Q}$, which in particular also does not matter how risk-averse the agent is. These phenomena remind us to check if the measure $\mathbb{Q}$ is independent of the utility $U$. It turns out that under certain regularity conditions, the answer is positive. We produce a short proof for this in Appendix A.

\footnotetext{
${ }^{2}$ See Theorem 20 in Chapter III of Protter (1990).
} 
Remark 3.1. Intuitionally, the 'strange' result that the equilibrium prices are independent of the agent's utility is actually true since in some way, the essence of a stochastic discounted factor is a measure of the wealth level of the market, which is completely determined by the flow of uncertain information generated only by the Brownian motion instead of the jump part of the dynamics defined in our model.

Based on what we discuss above, according to the dynamic asset pricing theory, see Duffie (2001) among others, we derive the following equilibrium price

$$
V_{t}^{\zeta}=\mathbb{E}^{\mathbb{Q}}\left[\int_{t}^{\infty} \exp (-r(s-t)) \zeta_{s} d s \mid \mathcal{F}_{t}\right], \quad t \in[0, \infty)
$$

for any asset defined by an $\mathbb{F}$-adapted stochastic process $\zeta$, which is the cash flow generated by the asset. In corporate finance, we often need to consider the pricing of the following asset: The cash flow rate of the asset is a linear homogeneous function of the cash flow of the firm, i.e. $\zeta_{t}=f\left(\delta_{t}\right)=a_{1} \delta_{t}+a_{2}, t \in[0, \infty)$ with $a_{1}$ and $a_{2}$ being constant, up to a stopping time $\tau_{\mathcal{D}} \equiv \inf \left\{t: \delta_{t} \notin \mathcal{D}\right\}$, which is the time of first departure of $\delta_{t}$ from a given domain $\mathcal{D}$. At the stopping time $\tau_{\mathcal{D}}$, the asset generates a lump-sum dividend, which is a function, denoted by $g(\cdot)$, of the cash flow rate $\delta_{\tau_{\mathcal{D}}} \notin \mathcal{D}$. After time $\tau_{\mathcal{D}}$, the asset disappears or its cash flow rate is zero forever. For the same reason with (7), the equilibrium price (value) of the asset is independent of time and given by

$$
\mathbb{E}^{\mathbb{Q}}\left[\int_{t}^{\tau_{\mathcal{D}}} \exp (-r(s-t))\left(a_{1} \delta_{s}+a_{2}\right) d s+e^{-r\left(\tau_{D}-t\right)} g\left(\delta_{\tau_{D}}\right) \mid \mathcal{F}_{t}\right]=V\left(\delta_{t}\right),
$$

for $\delta_{t} \in \mathcal{D}$ and some function $V(\cdot)$. Therefore, we conclude for $x \in \mathcal{D}$ that the function $V(\cdot)$ satisfies

$\frac{1}{2} \sigma^{2} V_{x x}(x)+\mu V_{x}(x)+\left(a_{1} x+a_{2}\right)+\lambda \int_{-\infty}^{+\infty}[V(x+z)-V(x)] h(z) d z-r V(x)=0$,

with the following condition:

$$
V(x)=g(x), \quad x \notin \mathcal{D},
$$

where the subscript of the function $V(\cdot)$ represents the differentiation with that variable. We provide a proof for (9) and (10) in Appendix B.

Before solving (9), we present one conclusion derived by Kou and Wang (2003) that introduces four numbers we will cite in the following text. The 
conclusion says that the equation

$$
G(\beta) \equiv \frac{1}{2} \sigma^{2} \beta^{2}+\mu \beta+\lambda\left(\frac{p \eta_{1}}{\eta_{1}-\beta}+\frac{q \eta_{2}}{\eta_{2}+\beta}-1\right)=r
$$

has four roots denoted by $\beta_{1}, \beta_{2},-\beta_{3}$ and $-\beta_{4}$ satisfying

$$
0<\beta_{1}<\eta_{1}<\beta_{2}<\infty, \quad 0<\beta_{3}<\eta_{2}<\beta_{4}<\infty .
$$

Clearly, if we assume $\lambda=0$, then the equation $G(\beta)=r$ has only two different roots, which are $\beta_{1}=\frac{-\beta+\sqrt{\beta^{2}+2 r \sigma^{2}}}{\sigma^{2}}$ and $-\beta_{3}=\frac{-\beta-\sqrt{\beta^{2}+2 r \sigma^{2}}}{\sigma^{2}}$.

If we assume $a_{1}=0$ and $\mathcal{D} \equiv(l, h)$, thanks to the Proposition 5.2. of Sepp (2004), the solution of (9) is given for $x \in(l, h)$ by

$$
V(x)=\bar{B}_{1} e^{\beta_{1}(x-h)}+\bar{B}_{2} e^{\beta_{2}(x-h)}+\bar{B}_{3} e^{-\beta_{3}(x-l)}+\bar{B}_{4} e^{-\beta_{4}(x-l)}+\frac{a_{2}}{r},
$$

where the parameters $\left\{\bar{B}_{i} ; i=1,2,3,4\right\}$ are to be determined from (10).

We note that the term $a_{2} / r$ in (11) is just the value of a perpetual bond with a fixed coupon of $a_{2}$. At the same time, we also note that the value of a claim at time $t \geq 0$ to the perpetual cash flow of $a_{1} \delta_{s}+a_{2}, s \in[t, \infty)$ is given from (6) by

$$
\begin{aligned}
& \mathbb{E}^{\mathbb{Q}}\left[\int_{t}^{\infty} e^{-r(s-t)}\left(a_{1} \delta_{s}+a_{2}\right) d s \mid \mathcal{F}_{t}\right] \\
& =a_{1} \int_{t}^{\infty} e^{-r(s-t)} \mathbb{E}^{\mathbb{Q}}\left[\delta_{s} \mid \mathcal{F}_{t}\right] d s+\frac{a_{2}}{r}=\frac{a_{1} x+a_{2}}{r}+a_{1} \frac{\mu+\lambda \xi}{r^{2}} .
\end{aligned}
$$

Taking both into consideration, ${ }^{3}$ if $a_{1} \neq 0$ and $x \in \mathcal{D} \equiv(l, h)$, we naturally guess that the solution of (9) would be given by

$$
\begin{aligned}
V(x) & =\bar{A}_{1} e^{\beta_{1}(x-h)}+\bar{A}_{2} e^{\beta_{2}(x-h)}+\bar{A}_{3} e^{-\beta_{3}(x-l)}+\bar{A}_{4} e^{-\beta_{4}(x-l)} \\
& +\frac{a_{1} x+a_{2}}{r}+a_{1} \frac{\mu+\lambda \xi}{r^{2}},
\end{aligned}
$$

where all the parameters $\left\{\bar{A}_{i} ; i=1,2,3,4\right\}$ are derived from (10). Actually, substituting (11) into (9), we verify after some tedious algebra that if the parameters $\left\{\bar{A}_{i} ; i=1,2,3,4\right\}$ are determined by (10), the function defined by (11) is really a solution of (9) with (10).

Remark 3.2. The two expressions (11) and (11) are powerful to derive the equilibrium prices of corporate securities as shown in the following subsection.

\footnotetext{
${ }^{3}$ In fact, each term in the guessed solution (11) has an economic explanation and if we follow these explanation, it is even easier for one to make such guess like us.
} 
3.2. Equilibrium prices of corporate securities and guarantee costs

Based on the above-stated equilibrium pricing theory, we explicitly derive all the prices of the contingent claims defined in Section 2 in this subsection. No matter whether the swap is EGS or OGS, naturally, it is not necessary for creditors to have a protective covenant. After taking into account that our model is a time-homogeneous Markov system, therefore, equityholders declare default at a stopping time $\tau_{\mathcal{D}}=\inf \left\{s \geq t: \delta_{s} \notin \mathcal{D} \equiv\left(\delta_{B},+\infty\right)\right\}$, where $\delta_{B}$ is a default threshold to be determined. Specifically, the equityholders must solve the following maximum-equity-valuation problem:

$$
E\left(\delta_{t}\right) \equiv \sup _{\delta_{B} \in \Re} \mathbb{E}^{\mathbb{Q}}\left[\int_{t}^{\tau_{\mathcal{D}}} e^{-r(s-t)}\left(1-\tau_{f}\right)\left(\delta_{s}-b\right) d s \mid \mathcal{F}_{t}\right]
$$

where $\tau_{f}$ is the effective tax rate derived from $1-\tau_{f}=\left(1-\tau_{c}\right)\left(1-\tau_{d}\right)$. According to (9) and (10), for $x \in \mathcal{D}$, the value function $E(\cdot)$ of equity satisfies:

$$
\begin{aligned}
& \mu E_{x}(x)+\frac{1}{2} \sigma^{2} E_{x x}(x)+\left(1-\tau_{f}\right)(x-b) \\
& +\lambda \int_{-\infty}^{+\infty}[E(x+z)-E(x)] f(z) d z=r E(x),
\end{aligned}
$$

with the condition

$$
E(x)=0, \quad x \notin \mathcal{D}
$$

and the following smooth-pasting condition since $\delta_{B}$ is an free boundary and determined by solving the maximum-equity-valuation problem

$$
E_{x}\left(\delta_{B}\right)=0
$$

According to (11), the solution is given by

$E(x)=P_{1} e^{-\beta_{3}\left(x-\delta_{B}\right)}+P_{2} e^{-\beta_{4}\left(x-\delta_{B}\right)}+\frac{\left(1-\tau_{f}\right) x}{r}-\frac{\left(1-\tau_{f}\right) b}{r}+\frac{\left(1-\tau_{f}\right)(\mu+\lambda \xi)}{r^{2}}$.

From (13), (14) and (16), we derive $P_{1}, P_{2}$ satisfying the following system of linear equations:

$$
\left\{\begin{array}{l}
\frac{P_{1} \eta_{2}}{\eta_{2}-\beta_{3}}+\frac{P_{2} \eta_{2}}{\eta_{2}-\beta_{4}}+\frac{\left(1-\tau_{f}\right) \delta_{B}}{r}-\frac{1-\tau_{f}}{r \eta_{2}}+\frac{\left(1-\tau_{f}\right)(\mu+\lambda \xi-r b)}{r^{2}}=0 \\
P_{1}+P_{2}+\frac{\left(1-\tau_{f}\right) \delta_{B}}{r}+\frac{\left(1-\tau_{f}\right)(\mu+\lambda \xi-r b)}{r^{2}}=0 .
\end{array}\right.
$$


By inserting its solution into (16), the value of equity is given by

$$
\begin{aligned}
& E(x)=\left(1-\tau_{f}\right)\left[\frac{\eta_{2}-\beta_{3}}{r \eta_{2}\left(\beta_{3}-\beta_{4}\right)}\left(1-b \beta_{4}+\beta_{4} \delta_{B}-\frac{\beta_{4}}{\eta_{2}}+\frac{(\mu+\lambda \xi) \beta_{4}}{r}\right) e^{-\beta_{3}\left(x-\delta_{B}\right)}\right. \\
& \left.+\frac{\eta_{2}-\beta_{4}}{r \eta_{2}\left(\beta_{4}-\beta_{3}\right)}\left(1-b \beta_{3}+\beta_{3} \delta_{B}-\frac{\beta_{3}}{\eta_{2}}+\frac{(\mu+\lambda \xi) \beta_{3}}{r}\right) e^{-\beta_{4}\left(x-\delta_{B}\right)}+\frac{x}{r}-\frac{b}{r}+\frac{\mu+\lambda \xi}{r^{2}}\right],
\end{aligned}
$$

and according to (15) and (17), the optimal default-triggering level $\delta_{B}$ is given by

$$
\delta_{B}=b-\frac{\mu+\lambda \xi}{r}-\frac{1}{\beta_{3}}-\frac{1}{\beta_{4}}+\frac{1}{\eta_{2}}
$$

To derive the value of equity under OGS, we must take into account the guarantee cost and optimal exercising strategy of the holder of the call option and therefore, we postpone the derivation later and now turn to the value of the option.

If an SME enters into OGS instead of EGS and the perpetual call option is not exercised, the optimal default-triggering level, denoted by $\delta_{\bar{B}}$, is, though, somewhat different from $\delta_{B}$ given by (18). It is in fact much more difficult to determine $\delta_{\bar{B}}$ than $\delta_{B}$. This work will be done only after the value of equity under OGS is derived and so it is also delayed.

For the perpetual American call option, due to the same reason of the time-homogeneous system, we conclude that at any time $t \geq 0$, there is a constant exercising boundary $\delta_{K}$ such that, once the cash flow rate $\delta$ hits the boundary $\delta_{K}$, the holder (insurer) will optimally exercise the perpetual American call option immediately. That is, for a given default threshold $\delta_{\bar{B}}$ before the option is exercised, which will be determined later, at any time $t \geq 0$ and $\delta_{t} \in \overline{\mathcal{D}} \equiv\left(\delta_{\bar{B}}, \delta_{K}\right)$, the value of the perpetual American call option is given by

$$
V^{A C}\left(\delta_{t}\right)=\max _{\delta_{K}}\left\{F\left(\delta_{\bar{B}}, \delta_{K}, \delta_{t}\right) \equiv \mathbb{E}^{\mathbb{Q}}\left[e^{-r\left(\tau_{\bar{D}}-t\right)} g\left(\delta_{\tau_{\bar{D}}}\right) \mid \mathcal{F}_{t}\right]\right\}
$$

where $\tau_{\bar{D}}=\inf \left\{s \geq t: \delta_{s} \notin \overline{\mathcal{D}}\right\}$ and the function $g(\cdot)$ is given by

$$
g(x)= \begin{cases}{[E(x)-K]^{+},} & x \geq \delta_{K} \\ 0, & x \leq \delta_{\bar{B}}\end{cases}
$$

where $E(x)$ is given by (17). 
According to $(8) \sim(10)$, the function $F\left(\delta_{\bar{B}}, \delta_{K}, \cdot\right)$ satisfies

$$
\begin{aligned}
& \mu F_{x}\left(\delta_{\bar{B}}, \delta_{K}, x\right)+\frac{\sigma^{2}}{2} F_{x x}\left(\delta_{\bar{B}}, \delta_{K}, x\right) \\
& +\lambda \int_{-\infty}^{+\infty}\left[F\left(\delta_{\bar{B}}, \delta_{K}, x+z\right)-F\left(\delta_{\bar{B}}, \delta_{K}, x\right)\right] f(z) d z-r F\left(\delta_{\bar{B}}, \delta_{K}, x\right)=0
\end{aligned}
$$

with the conditions

$$
F\left(\delta_{\bar{B}}, \delta_{K}, x\right)= \begin{cases}{[E(x)-K]^{+},} & x \geq \delta_{K} \\ 0, & x \leq \delta_{\bar{B}}\end{cases}
$$

Thanks to (11), when $x \in \overline{\mathcal{D}}$, the function $F\left(\delta_{\bar{B}}, \delta_{K}, \cdot\right)$ is given by

$F\left(\delta_{\bar{B}}, \delta_{K}, x\right)=B_{1} e^{\beta_{1}\left(x-\delta_{K}\right)}+B_{2} e^{\beta_{2}\left(x-\delta_{K}\right)}+B_{3} e^{-\beta_{3}\left(x-\delta_{\bar{B}}\right)}+B_{4} e^{-\beta_{4}\left(x-\delta_{\bar{B}}\right)}$.

From (19), (20) and (21), we conclude that the parameters $B_{1}, B_{2}, B_{3}$ and $B_{4}$ satisfy the following system of linear equations:

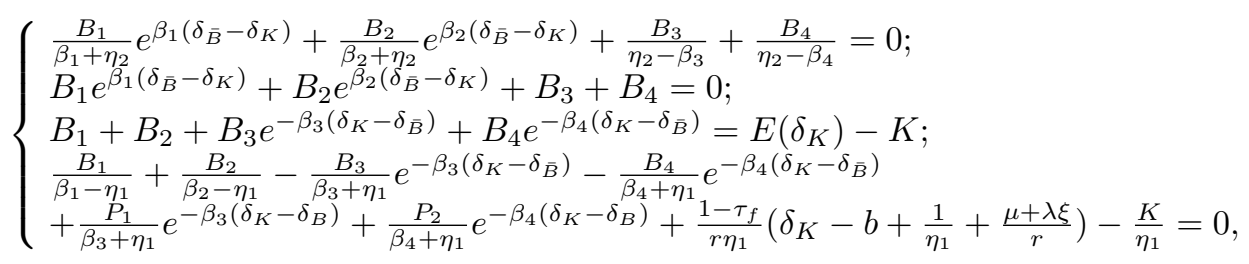

where $E\left(\delta_{K}\right)$ is given by (17).

The optimal exercising boundary $\delta_{K}$ is given by the following smoothpasting condition:

$$
\left.\frac{\partial F\left(\delta_{\bar{B}}, \delta_{K}, x\right)}{\partial x}\right|_{x=\delta_{K}}=\left.\frac{\partial E(x)}{\partial x}\right|_{x=\delta_{K}} .
$$

To sum up, at any time $t \geq 0$ and $\delta_{t} \in\left(\delta_{\bar{B}}, \delta_{K}\right)$, the value $V^{A C}\left(\delta_{t}\right)$ of the perpetual American call option held by the insurer in the option-forguarantee swap is given by

$$
V^{A C}\left(\delta_{t}\right)=B_{1} e^{\beta_{1}\left(\delta_{t}-\delta_{K}\right)}+B_{2} e^{\beta_{2}\left(\delta_{t}-\delta_{K}\right)}+B_{3} e^{-\beta_{3}\left(\delta_{t}-\delta_{\bar{B}}\right)}+B_{4} e^{-\beta_{4}\left(\delta_{t}-\delta_{\bar{B}}\right)},
$$

where the optimal exercising boundary $\delta_{K}$ is derived from (21) and (22). It is clear that under a given coupon payment $b$, the value of debt is completely determined by default threshold. As shown above, we have two different default thresholds: $\delta_{B}$ and $\delta_{\bar{B}}$. For this reason, we consider the value of 
debt, denoted by $D\left(\delta_{B}, \delta_{t}\right)$, under the case of that an SME enters into EGS or OGS but the option is exercised, i.e $\delta_{B}$ is the default threshold and one, denoted by $D\left(\delta_{\bar{B}}, \delta_{K}, \delta_{B}, \delta_{t}\right)$, under the case of that an SME enters into OGS and the option is not exercised, i.e. $\delta_{\bar{B}}$ is the default threshold.

First, we have

$$
D\left(\delta_{B}, \delta_{t}\right)=\mathbb{E}^{\mathbb{Q}}\left[\int_{t}^{\tau_{\mathcal{D}}} \exp (-r(s-t))\left(1-\tau_{i}\right) b d s+e^{-r\left(\tau_{\mathcal{D}}-t\right)} g\left(\delta_{\tau_{\mathcal{D}}}\right) \mid \mathcal{F}_{t}\right],
$$

Thanks to the equilibrium pricing theory shown in subsection 3.1, we get that for $x \in \mathcal{D}=\left(\delta_{B}, \infty\right)$, the function $D\left(\delta_{B}, \cdot\right)$ satisfies

$$
\begin{gathered}
\mu D_{x}\left(\delta_{B}, x\right)+\frac{1}{2} \sigma^{2} D_{x x}\left(\delta_{B}, x\right)+\left(1-\tau_{i}\right) b \\
+\lambda \int_{-\infty}^{+\infty}\left[D\left(\delta_{B}, x+z\right)-D\left(\delta_{B}, x\right)\right] f(z) d z=r D\left(\delta_{B}, x\right)
\end{gathered}
$$

with the condition

$$
D\left(\delta_{B}, x\right)=\left(1-\tau_{f}\right)(1-\alpha) \frac{\mu+\lambda \xi+r x}{r^{2}} \quad x \leq \delta_{B},
$$

where $\alpha$ is the bankruptcy loss rate. The bankruptcy loss can be interpreted as loss from selling the real assets, asset fire-sale losses, legal fees, etc. According to (11), the solution is given by

$$
D\left(\delta_{B}, \delta_{t}\right)=\tilde{A} e^{-\beta_{3}\left(\delta_{t}-\delta_{B}\right)}+\tilde{B} e^{-\beta_{4}\left(\delta_{t}-\delta_{B}\right)}+\frac{\left(1-\tau_{i}\right) b}{r},
$$

From $(24),(24),(26)$ and $(27)$, we can get $\tilde{A}$ and $\tilde{B}$ satisfy the following system of linear equations:

$$
\left\{\begin{array}{l}
\tilde{A}+\tilde{B}=\left(1-\tau_{f}\right)(1-\alpha) \frac{\mu+\lambda \xi+r \delta_{B}}{r^{2}}-\frac{\left(1-\tau_{i}\right) b}{r}, \\
\frac{\tilde{A}}{\eta_{2}-\beta_{3}}+\frac{\tilde{B}}{\eta_{2}-\beta_{4}}=-\frac{\left(1-\tau_{i}\right) b}{r \eta_{2}}+\frac{\left(1-\tau_{f}\right)(1-\alpha)}{r \eta_{2}}\left(\delta_{B}-\frac{1}{\eta_{2}}+\frac{\mu+\lambda \xi}{r}\right) .
\end{array}\right.
$$

Thus, for $\delta_{t} \in\left(\delta_{B}, \infty\right)$, we get the value of debt under EGS as follows:

$$
\begin{aligned}
& D\left(\delta_{B}, \delta_{t}\right) \\
& =\frac{\eta_{2}-\beta_{3}}{\left(\beta_{4}-\beta_{3}\right) r \eta_{2}}\left[\left(1-\tau_{f}\right)(1-\alpha)\left(\frac{\left(\mu+\lambda \xi+r \delta_{B}\right) \beta_{4}}{r}+\frac{\eta_{2}-\beta_{4}}{\eta_{2}}\right)-\left(1-\tau_{i}\right) b \beta_{4}\right] e^{-\beta_{3}\left(\delta_{t}-\delta_{B}\right)} \\
& \quad+\frac{\eta_{2}-\beta_{4}}{\left(\beta_{4}-\beta_{3}\right) r \eta_{2}}\left[\left(1-\tau_{i}\right) b \beta_{3}-\left(1-\tau_{f}\right)(1-\alpha)\left(\frac{\left(\mu+\lambda \xi+r \delta_{B}\right) \beta_{3}}{r}+\frac{\eta_{2}-\beta_{3}}{\eta_{2}}\right)\right] e^{-\beta_{4}\left(\delta_{t}-\delta_{B}\right)} \\
& \quad+\frac{\left(1-\tau_{i}\right) b}{r} .
\end{aligned}
$$


Second, following (8) (10), we derive the value of debt under OGS and before the option is exercised as follows:

$D\left(\delta_{\bar{B}}, \delta_{K}, \delta_{B}, \delta_{t}\right)=\mathbb{E}^{\mathbb{Q}}\left[\int_{t}^{\tau_{\overline{\mathcal{D}}}} \exp (-r(s-t))\left(1-\tau_{i}\right) b d s+e^{-r\left(\tau_{\overline{\mathcal{D}}}-t\right)} g\left(\delta_{\tau_{\overline{\mathcal{D}}}}\right) \mid \mathcal{F}_{t}\right]$,

where $\overline{\mathcal{D}}=\left(\delta_{\bar{B}}, \delta_{K}\right)$ and

$$
g(x)= \begin{cases}\left(1-\tau_{f}\right)(1-\alpha) \frac{\mu+\lambda \xi+r x}{r^{2}}, & x \leq \delta_{\bar{B}} \\ D\left(\delta_{B}, x\right) & x \geq \delta_{K}\end{cases}
$$

After that, the function $D\left(\delta_{\bar{B}}, \delta_{K}, \delta_{B}, \cdot\right)$ satisfies

$$
\begin{aligned}
& \mu D_{x}\left(\delta_{\bar{B}}, \delta_{K}, \delta_{B}, x\right)+\frac{1}{2} \sigma^{2} D_{x x}\left(\delta_{\bar{B}}, \delta_{K}, \delta_{B}, x\right)+\left(1-\tau_{i}\right) b \\
& +\lambda \int_{-\infty}^{+\infty}\left[D\left(\delta_{\bar{B}}, \delta_{K}, \delta_{B}, x+z\right)-D\left(\delta_{\bar{B}}, \delta_{K}, \delta_{B}, x\right)\right] f(z) d z=r D\left(\delta_{\bar{B}}, \delta_{K}, \delta_{B}, x\right),
\end{aligned}
$$

with the boundary condition

$$
\begin{cases}D\left(\delta_{\bar{B}}, \delta_{K}, \delta_{B}, x\right)=\left(1-\tau_{f}\right)(1-\alpha) \frac{\mu+\lambda \xi+r x}{r^{2}}, & x \leq \delta_{\bar{B}} \\ D\left(\delta_{\bar{B}}, \delta_{K}, \delta_{B}, x\right)=D\left(\delta_{B}, x\right), & x \geq \delta_{K}\end{cases}
$$

According to (11), the solution is given by

$$
\begin{aligned}
& D\left(\delta_{\bar{B}}, \delta_{K}, \delta_{B}, \delta_{t}\right) \\
& =C_{1} e^{\beta_{1}\left(\delta_{t}-\delta_{K}\right)}+C_{2} e^{\beta_{2}\left(\delta_{t}-\delta_{K}\right)}+C_{3} e^{-\beta_{3}\left(\delta_{t}-\delta_{\bar{B}}\right)}+C_{4} e^{-\beta_{4}\left(\delta_{t}-\delta_{\bar{B}}\right)}+\frac{\left(1-\tau_{i}\right) b}{r},
\end{aligned}
$$

where $C_{i}, i=1,2,3,4$ are constants to be determined. From (28), (29), (30) and (31), we conclude that $C_{1}, C_{2}, C_{3}$ and $C_{4}$ satisfy the following system of linear equations:

$$
\left\{\begin{array}{l}
\frac{C_{1}}{\beta_{1}+\eta_{2}} e^{\beta_{1}\left(\delta_{\bar{B}}-\delta_{K}\right)}+\frac{C_{2}}{\beta_{2}+\eta_{2}} e^{\beta_{2}\left(\delta_{\bar{B}}-\delta_{K}\right)}+\frac{C_{3}}{\eta_{2}-\beta_{3}}+\frac{C_{4}}{\eta_{2}-\beta_{4}} \\
+\frac{\left(1-\tau_{i}\right) b}{r \eta_{2}}-\frac{\left(1-\tau_{f}\right)(1-\alpha)}{r \eta_{2}}\left(\frac{\mu+\lambda \xi+r \delta_{\bar{B}}}{r}-\frac{1}{\eta_{2}}\right)=0 \\
\frac{C_{1}}{\beta_{1}-\eta_{1}}+\frac{C_{2}}{\beta_{2}-\eta_{1}}-\frac{C_{3}}{\beta_{3}+\eta_{1}} e^{-\beta_{3}\left(\delta_{K}-\delta_{\bar{B}}\right)}-\frac{C_{4}}{\beta_{4}+\eta_{1}} e^{-\beta_{4}\left(\delta_{K}-\delta_{\bar{B}}\right)} \\
+\frac{\tilde{A}}{\beta_{3}+\eta_{1}} e^{-\beta_{3}\left(\delta_{K}-\delta_{B}\right)}+\frac{\tilde{B}}{\beta_{4}+\eta_{1}} e^{-\beta_{4}\left(\delta_{K}-\delta_{B}\right)}=0 \\
C_{1} e^{\beta_{1}\left(\delta_{\bar{B}}-\delta_{K}\right)}+C_{2} e^{\beta_{2}\left(\delta_{\bar{B}}-\delta_{K}\right)}+C_{3}+C_{4}+\frac{\left(1-\tau_{i}\right) b}{r}=\left(1-\tau_{f}\right)(1-\alpha) \frac{\mu+\lambda \xi+r \delta_{\bar{B}}}{r^{2}} \\
C_{1}+C_{2}+C_{3} e^{-\beta_{3}\left(\delta_{K}-\delta_{\bar{B}}\right)}+C_{4} e^{-\beta_{4}\left(\delta_{K}-\delta_{\bar{B}}\right)}=\tilde{A} e^{-\beta_{3}\left(\delta_{K}-\delta_{B}\right)}+\tilde{B} e^{-\beta_{4}\left(\delta_{K}-\delta_{B}\right)}
\end{array}\right.
$$

Now, we are ready to determine how much guarantee cost an SME should be charged for getting the loan. 
Naturally, we assume the present time is $t=0$ when the swap contract is signed and $\delta_{0} \in \mathcal{D}=\left(\delta_{B}, \infty\right)$ under EGS, while $\delta_{0} \in \overline{\mathcal{D}}=\left(\delta_{\bar{B}}, \delta_{K}\right)$ under OGS. In the following, we denote by $V^{e}\left(\delta_{0}\right)$ and $V^{o}\left(\delta_{0}\right)$ the equilibrium value of the insurer's contingent compensatory payment to the bank/lender under EGS and OGS respectively, which are taxed at a personal rate $\tau_{i}$.

Clearly, under EGS, in order to fully protect the lender, the value $V^{e}\left(\delta_{0}\right)$ must satisfy the following equality:

$$
D\left(\delta_{B}, \delta_{0}\right)+\left(1-\tau_{i}\right) V^{e}\left(\delta_{0}\right)=\frac{b}{r}\left(1-\tau_{i}\right) .
$$

From (27), we obtain that the value $V^{e}\left(\delta_{0}\right)$ of the insurer's compensatory payment to the bank/lender is given by

$$
V^{e}\left(\delta_{0}\right)=-\frac{\tilde{A} e^{-\beta_{3}\left(\delta_{0}-\delta_{B}\right)}+\tilde{B} e^{-\beta_{4}\left(\delta_{0}-\delta_{B}\right)}}{1-\tau_{i}} .
$$

Consequently, in order to make the EGS contract fair, i.e. make sure that the value of what an insurer pays is equal to the value of the cash flow the insurer gets due to the guarantee, we must have from (32) that

$$
V^{e}\left(\delta_{0}\right)=\varphi^{e} E\left(\delta_{0}\right)
$$

where $\varphi^{e}$ is called guarantee cost, i.e. the amount (fraction) of equity allocated to the insurer in return for his guarantee.

Therefore, we derive from (33) the following explicit guarantee cost:

$$
\varphi^{e}=-\frac{\tilde{A} e^{-\beta_{3}\left(\delta_{0}-\delta_{B}\right)}+\tilde{B} e^{-\beta_{4}\left(\delta_{0}-\delta_{B}\right)}}{\left(1-\tau_{i}\right) E\left(\delta_{0}\right)},
$$

where $E\left(\delta_{0}\right)$ is given by (17). Naturally, the value, denoted by $E^{e}\left(\delta_{t}, \varphi^{e}\right)$, of equity held by a firm who enters into EGS is given by

$$
E^{e}\left(\delta_{t}, \varphi^{e}\right)=\left(1-\varphi^{e}\right) E\left(\delta_{t}\right)
$$

In the same way, when the contract is an option-for-guarantee swap (OGS), in order to fully protect the lender, the value $V^{o}\left(\delta_{0}\right)$ of the insurer's contingent compensatory payment must satisfy the following equality:

$$
D\left(\delta_{\bar{B}}, \delta_{K}, \delta_{B}, \delta_{0}\right)+\left(1-\tau_{i}\right) V^{o}\left(\delta_{0}\right)=\frac{b}{r}\left(1-\tau_{i}\right) .
$$

Then, utilizing (31), we get the value $V^{o}\left(\delta_{0}\right)$ as follows:

$V^{o}\left(\delta_{0}\right)=-\frac{C_{1} e^{\beta_{1}\left(\delta_{0}-\delta_{K}\right)}+C_{2} e^{\beta_{2}\left(\delta_{0}-\delta_{K}\right)}+C_{3} e^{-\beta_{3}\left(\delta_{0}-\delta_{\bar{B}}\right)}+C_{4} e^{-\beta_{4}\left(\delta_{0}-\delta_{\bar{B}}\right)}}{1-\tau_{i}}$. 
Consequently, in order to make the swap contract fair, we must have from (34) that

$$
V^{o}\left(\delta_{0}\right)=\varphi^{o} V^{A C}\left(\delta_{0}\right)
$$

where $\varphi^{o}$ is called guarantee cost, i.e. the amount (fraction) of the American call option held by the insurer in return for the guarantee.

Therefore, we derive from (35) the following explicit guarantee cost:

$$
\varphi^{o}=-\frac{C_{1} e^{\beta_{1}\left(\delta_{0}-\delta_{K}\right)}+C_{2} e^{\beta_{2}\left(\delta_{0}-\delta_{K}\right)}+C_{3} e^{-\beta_{3}\left(\delta_{0}-\delta_{\bar{B}}\right)}+C_{4} e^{-\beta_{4}\left(\delta_{0}-\delta_{\bar{B}}\right)}}{\left(1-\tau_{i}\right) V^{A C}\left(\delta_{0}\right)},
$$

where $V^{A C}\left(\delta_{0}\right)$ is given by (23).

Last, we turn to the value of equity under OGS and the default-triggering level $\delta_{\bar{B}}$ selected by an SME, who enters into OGS and aims to maximize the equilibrium value of his claim before the option is exercised.

For this aim, there are two different expressions of the value of equity: One is denoted by $\bar{E}^{o}\left(\delta_{t}, \varphi^{o}\right)$, which corresponds to the case that the option is not exercised; The other is denoted by $E^{o}\left(\delta_{t}, \varphi^{o}\right)$, which corresponds to the case that the option has been exercised.

First, we note that under any given OGS contract, the guarantee cost must be determined at the initial time when the contract is signed. Therefore, if the option has been exercised, and the firm has not gone bankrupt, we conclude directly from (17) that the value $E^{o}\left(\delta_{t}, \varphi^{o}\right)$ is given by

$$
\begin{aligned}
E^{o}\left(\delta_{t}, \varphi^{o}\right) \\
=\left(1-\tau_{f}\right)\left(1-\varphi^{o}\right)\left[\frac{\eta_{2}-\beta_{3}}{r \eta_{2}\left(\beta_{3}-\beta_{4}\right)}\left(1-b \beta_{4}+\beta_{4} \delta_{B}-\frac{\beta_{4}}{\eta_{2}}+\frac{(\mu+\lambda \xi) \beta_{4}}{r}\right) e^{-\beta_{3}\left(\delta_{t}-\delta_{B}\right)}\right. \\
\left.\quad+\frac{\eta_{2}-\beta_{4}}{r \eta_{2}\left(\beta_{4}-\beta_{3}\right)}\left(1-b \beta_{3}+\beta_{3} \delta_{B}-\frac{\beta_{3}}{\eta_{2}}+\frac{(\mu+\lambda \xi) \beta_{3}}{r}\right) e^{-\beta_{4}\left(\delta_{t}-\delta_{B}\right)}+\frac{\delta_{t}}{r}-\frac{b}{r}+\frac{\mu+\lambda \xi}{r^{2}}\right],
\end{aligned}
$$

where the default threshold $\delta_{B}$ is given by (18).

Second, if the option has not been exercised and the firm has not gone bankrupt, i.e. $\delta_{t} \in \overline{\mathcal{D}} \equiv\left(\delta_{\bar{B}}, \delta_{K}\right)$, the equilibrium value $\bar{E}^{o}\left(\delta_{t}, \varphi^{o}\right)$ of the SME's claim is given by

$$
\bar{E}^{o}\left(\delta_{t}, \varphi^{o}\right)=\sup _{\delta_{\bar{B}} \in \Re} \mathbb{E}^{\mathbb{Q}}\left[\int_{t}^{\tau_{\bar{D}}} e^{-r(s-t)}\left(1-\tau_{f}\right)\left(\delta_{s}-b\right) d s+e^{-r\left(\tau_{\bar{D}}-t\right)} g\left(\delta_{\tau_{\bar{D}}}\right) \mid \mathcal{F}_{t}\right],
$$

where according to the OGS agreement, the function $g(\cdot)$ is defined by

$$
g(x)= \begin{cases}0, & x \leq \delta_{\bar{B}} \\ E^{o}\left(x, \varphi^{o}\right)+\varphi^{o} K, & x \geq \delta_{K} .\end{cases}
$$

Therefore, utilizing the equilibrium pricing theory provided in subsection 3.1 and a standard endogenous default timing theory, we obtain that the 
market value $\bar{E}^{o}\left(x, \varphi^{o}\right)$ of equity satisfies the following differential equation:

$$
\begin{aligned}
& \mu \bar{E}_{x}^{o}+\frac{\sigma^{2}}{2} \bar{E}_{x x}^{o}+\left(1-\tau_{f}\right)(x-b) \\
& +\lambda \int_{-\infty}^{+\infty}\left[\bar{E}^{o}(x+z)-\bar{E}^{o}(x)\right] f(z) d z=r \bar{E}^{o}, \quad \delta_{\bar{B}}<x<\delta_{K}
\end{aligned}
$$

with the following value-matching and smooth-pasting conditions ${ }^{4}$

$$
\begin{cases}\bar{E}^{o}\left(x, \varphi^{o}\right)=E^{o}\left(x, \varphi^{o}\right)+\varphi^{o} K, & x \geq \delta_{K} \\ \bar{E}^{o}\left(x, \varphi^{o}\right)=0, & x \leq \delta_{\bar{B}} ; \\ \bar{E}_{x}^{o}\left(\delta_{\bar{B}}, \varphi^{o}\right)=0 . & \end{cases}
$$

According to (11), we get the following explicit solution of (36) with (37):

$$
\begin{aligned}
\bar{E}^{o}\left(x, \varphi^{o}\right)= & A_{1} e^{\beta_{1}\left(x-\delta_{K}\right)}+A_{2} e^{\beta_{2}\left(x-\delta_{K}\right)}+A_{3} e^{-\beta_{3}\left(x-\delta_{\bar{B}}\right)}+A_{4} e^{-\beta_{4}\left(x-\delta_{\bar{B}}\right)} \\
& +\frac{\left(1-\tau_{f}\right) x}{r}-\frac{\left(1-\tau_{f}\right) b}{r}+\frac{\left(1-\tau_{f}\right)(\mu+\lambda \xi)}{r^{2}},
\end{aligned}
$$

where $\left\{A_{i} ; i=1,2,3,4\right\}$ are constants to be determined. From (36) (38), we conclude that $\left\{A_{i} ; i=1,2,3,4\right\}$ satisfy the following system of linear equations:

$$
\left\{\begin{array}{l}
\frac{A_{1}}{\beta_{1}+\eta_{2}} e^{\beta_{1}\left(\delta_{\bar{B}}-\delta_{K}\right)}+\frac{A_{2}}{\beta_{2}+\eta_{2}} e^{\beta_{2}\left(\delta_{\bar{B}}-\delta_{K}\right)}+\frac{A_{3}}{\eta_{2}-\beta_{3}}+\frac{A_{4}}{\eta_{2}-\beta_{4}} \\
+\frac{1-\tau_{f}}{r \eta_{2}}\left(\frac{\mu+\lambda \xi}{r}-\frac{1}{\eta_{1}}+\delta_{\bar{B}}-b\right)=0, \\
\frac{A_{1} \eta_{1}}{\beta_{1}-\eta_{1}}+\frac{A_{2} \eta_{1}}{\beta_{2}-\eta_{1}}-\frac{A_{3} \eta_{1}}{\beta_{3}+\eta_{1}} e^{-\beta_{3}\left(\delta_{K}-\delta_{\bar{B}}\right)}-\frac{A_{4} \eta_{1}}{\beta_{4}+\eta_{1}} e^{-\beta_{4}\left(\delta_{K}-\delta_{\bar{B}}\right)} \\
+\frac{\left(1-\tau_{f}\right)\left(1-\varphi^{o}\right) \eta_{1}}{r \eta_{2}\left(\beta_{3}-\beta_{4}\right)}\left[\frac{\eta_{2}-\beta_{3}}{\beta_{3}+\eta_{1}}\left(1-b \beta_{4}+\beta_{4} \delta_{B}-\frac{\beta_{4}}{\eta_{2}}+\frac{(\mu+\lambda \xi) \beta_{4}}{r}\right) e^{-\beta_{3}\left(\delta_{K}-\delta_{B}\right)}\right. \\
\left.-\frac{\eta_{2}-\beta_{4}}{\beta_{4}+\eta_{1}}\left(1-b \beta_{3}+\beta_{3} \delta_{B}-\frac{\beta_{3}}{\eta_{2}}+\frac{(\mu+\lambda \xi) \beta_{3}}{r}\right) e^{-\beta_{4}\left(\delta_{K}-\delta_{B}\right)}\right] \\
-\frac{\left(1-\tau_{f}\right) \varphi^{o}}{r}\left(\delta_{K}-b+\frac{1}{\eta_{1}}+\frac{\mu+\lambda \xi}{r}\right)+\varphi^{o} K=0, \\
A_{1} e^{\beta_{1}\left(\delta_{\bar{B}}-\delta_{K}\right)}+A_{2} e^{\beta_{2}\left(\delta_{\bar{B}}-\delta_{K}\right)}+A_{3}+A_{4}+\frac{\left(1-\tau_{f}\right) \delta_{\bar{B}}}{r}+\frac{\left(1-\tau_{f}\right)(\mu+\lambda \xi)}{r^{2}}-\frac{\left(1-\tau_{f}\right) b}{r}=0, \\
A_{1}+A_{2}+A_{3} e^{-\beta_{3}\left(\delta_{K}-\delta_{\bar{B}}\right)}+A_{4} e^{-\beta_{4}\left(\delta_{K}-\delta_{\bar{B}}\right)}+\left(1-\tau_{f}\right)\left(\frac{\delta_{K}}{r}+\frac{\mu+\lambda \xi}{r^{2}}-\frac{b}{r}\right) \\
=\left(1-\tau_{f}\right)\left(1-\varphi^{o}\right)\left[\frac{\eta_{2}-\beta_{3}}{r \eta_{2}\left(\beta_{3}-\beta_{4}\right)}\left(1-b \beta_{4}+\beta_{4} \delta_{B}-\frac{\beta_{4}}{\eta_{2}}+\frac{(\mu+\lambda \xi) \beta_{4}}{r}\right) e^{-\beta_{3}\left(\delta_{K}-\delta_{B}\right)}\right. \\
\left.+\frac{\eta_{2}-\beta_{4}}{r \eta_{2}\left(\beta_{4}-\beta_{3}\right)}\left(1-b \beta_{3}+\beta_{3} \delta_{B}-\frac{\beta_{3}}{\eta_{2}}+\frac{(\mu+\lambda \xi) \beta_{3}}{r}\right) e^{-\beta_{4}\left(\delta_{K}-\delta_{B}\right)}+\frac{\delta_{K}-b}{r}+\frac{\mu+\lambda \xi}{r^{2}}\right]+\varphi^{o} K,
\end{array}\right.
$$

and the default threshold $\delta_{\bar{B}}$ is a solution of the following equation

$$
\beta_{1} A_{1} e^{\beta_{1}\left(\delta_{\bar{B}}-\delta_{K}\right)}+\beta_{2} A_{2} e^{\beta_{2}\left(\delta_{\bar{B}}-\delta_{K}\right)}-\beta_{3} A_{3}-\beta_{4} A_{4}+\frac{1-\tau_{f}}{r}=0 .
$$

\footnotetext{
${ }^{4}$ The exercising boundary $\delta_{K}$ in (37) is determined in advance and therefore, the smooth-pasting condition at the point $\delta_{K}$ is not imposed here.
} 
Remark 3.3. To solve the simultaneous equations defined by (18), (22) and (39), we are able to get the optimal default threshold $\delta_{B}$ after the option is exercised, the default threshold $\delta_{\bar{B}}$ before the option is exercised and the optimal exercising boundary $\delta_{K}$ simultaneously. Clearly, the strategy profile determined by the default thresholds $\delta_{B}$ and $\delta_{\bar{B}}$ selected by an SME and an exercising boundary $\delta_{K}$ selected by an insurer is actually a Nash equilibrium of the game between the SME and the insurer from game theory.

\section{COMPARATIVE STATICS AND NUMERICAL RESULTS}

In this section, we examine how default thresholds, the exercising boundary of the option, guarantee costs and the value of equity held by an SME who enters into EGS or OGS change with cash flow volatility $\sigma$, correlation $\rho$ and jump intensity $\lambda$ respectively. To this end, all model parameters take the following annualized baseline parameter values unless otherwise stated: the risk-free interest rate $r=0.05$, the expected growth rate of the cash flow $\mu_{\delta}=0.2$, cash flow volatility $\sigma=0.9$, initial cash flow rate $\delta_{0}=10$ correlation coefficient $\rho=0.2$ between the market and the cash flow, market Sharpe ratio $\eta=0.4$, coupon $b=8$, exercise price $K=35$, bankruptcy loss rate $\alpha=0.5$, jump intensity $\lambda=0.5$, the probabilities $p=0.4(q=0.6)$ of upward (downward) jumps, and the means of the two exponential distributions $\frac{1}{\eta_{1}}=\frac{1}{3}, \frac{1}{\eta_{2}}=\frac{1}{4}$. According to the Tax System of China, personal rate $\tau_{i}=0.05$, corporate profit tax rate $\tau_{c}=0.25$, effective dividend tax rate $\tau_{d}=0.2$ and so the effective tax rate $\tau_{f}=0.4$ derived from $1-\tau_{f}=\left(1-\tau_{c}\right)\left(1-\tau_{d}\right)$.

4.1. Equilibrium prices vs. the diffusive volatility of the cash flow

TABLE 1.

The table represents the impacts of changes in the diffusive volatility $(\sigma)$ of cash flow with the baseline parameter values.

\begin{tabular}{|l|l|l|l|l|}
\hline$\sigma$ & 0.3 & 0.5 & 0.7 & 0.9 \\
\hline$\delta_{B}$ & 4.2526 & 4.1462 & 3.9141 & 3.5992 \\
\hline$\delta_{\bar{B}}$ & 6.5875 & 6.4850 & 6.2650 & 5.9750 \\
\hline$\delta_{K}$ & 11.3150 & 11.7275 & 12.2275 & 12.7575 \\
\hline$\varphi^{e}$ & $1.2072 \mathrm{e}-5$ & 0.0020 & 0.0259 & 0.0949 \\
\hline$\varphi^{o}$ & 0.0022 & 0.0496 & 0.2310 & 0.5104 \\
\hline$E^{e}\left(\delta_{0}, \varphi^{e}\right)$ & 64.2393 & 60.2912 & 55.2987 & 48.6310 \\
\hline $\bar{E}^{o}\left(\delta_{0}, \varphi^{o}\right)$ & 64.1548 & 58.6867 & 49.5662 & 39.0529 \\
\hline
\end{tabular}


Table 1 describes the impacts of the diffusive volatility $\sigma$ of the cash flow on optimal default thresholds $\delta_{B}, \delta_{\bar{B}}$, exercise boundary $\delta_{K}$, guarantee cost $\varphi^{e}$ under the EGS, guarantee $\operatorname{cost} \varphi^{o}$ under the OGS, equilibrium values $E^{e}\left(\delta_{0}, \varphi^{e}\right)$ and $\bar{E}^{o}\left(\delta_{0}, \varphi^{o}\right)$ of equity held by the entrepreneur under EGS and under OGS respectively.

As we expected, Table 1 states that the two optimal default thresholds decrease with the diffusive volatility, i.e. the higher the business risk, the later the entrepreneur defaults. However, on the contrary to the well-known conclusion that the value of equity increases with the diffusive volatility, as the business risk (volatility $\sigma$ ) increases, the value of the equity held by the entrepreneur conversely decreases rather than goes up. This is because as business risk rises, the insurer's compensatory payment $V^{o}\left(\delta_{0}\right)\left(V^{e}\left(\delta_{0}\right)\right)$ ascends and naturally, the guarantee $\operatorname{cost} \varphi^{o}\left(\varphi^{e}\right)$ increase. Clearly, the larger the guarantee $\operatorname{cost} \varphi^{o}$ or $\varphi^{e}$, the less the amount of equity left to the entrepreneur and so the less the equilibrium values $E^{e}\left(\delta_{0}, \varphi^{e}\right)$ and $\bar{E}^{o}\left(\delta_{0}, \varphi^{o}\right)$ of equity held by the entrepreneur.

Table 1 shows that EGS is better than OGS for the entrepreneur while keeping the other two parties in the agreement the same. The bigger the volatility $\sigma$, the bigger the difference between the two equilibrium values $E^{e}\left(\delta_{0}, \varphi^{e}\right)$ and $\bar{E}\left(\delta_{0}, \varphi^{o}\right)$. The larger the volatility, the bigger the difference $\delta_{\bar{B}}-\delta_{B}>0$ and thus, the value of equity held by the entrepreneur under OGS moves downward faster than that under EGS since a bigger default threshold leads to a higher default probability and bankruptcy cost.

\subsection{Equilibrium prices vs. cash flow correlation with market portfolio}

TABLE 2.

The table represents the impacts of changes in correlation coefficient $(\rho)$ between the cash flow and the return of the market portfolio with the baseline parameter values.

\begin{tabular}{|l|l|l|l|l|}
\hline$\rho$ & -0.4 & -0.2 & 0 & 0.2 \\
\hline$\delta_{B}$ & 0.1632 & 1.3925 & 2.5479 & 3.5992 \\
\hline$\delta_{\bar{B}}$ & 2.4969 & 3.7279 & 4.8918 & 5.9705 \\
\hline$\delta_{K}$ & 12.0359 & 12.2295 & 12.4639 & 12.7557 \\
\hline$\varphi^{e}$ & $1.9195 \mathrm{e}-4$ & 0.0022 & 0.0175 & 0.0949 \\
\hline$\varphi^{o}$ & 0.0017 & 0.0154 & 0.1007 & 0.5093 \\
\hline$E^{e}\left(\delta_{0}, \varphi^{e}\right)$ & 104.5423 & 87.1112 & 68.9704 & 48.6310 \\
\hline $\bar{E}^{o}\left(\delta_{0}, \varphi^{o}\right)$ & 104.4218 & 86.3225 & 65.6245 & 39.0872 \\
\hline
\end{tabular}

Now we turn to the impacts of correlation $\rho$ between the firm's cash flow and the market portfolio plotted by Table 2 on default thresholds, exercise boundary, guarantee costs and equilibrium prices. 
Table 2 states that with a growth of correlation $\rho$, both default thresholds go up. This is because the larger the correlation $\rho$, the smaller the riskadjusted return $\mu=\mu_{\delta}-\rho \sigma \eta$ and therefore, the less the value of equity, which naturally leads to an earlier default. On the other hand, similar with Table 1, EGS is better than OGS for the entrepreneur while keeping the other two parties in the agreement the same. The bigger the correlation coefficient $\rho$, the bigger the difference between the two equilibrium values $E^{e}\left(\delta_{0}, \varphi^{e}\right)$ and $\bar{E}\left(\delta_{0}, \varphi^{o}\right)$.

\subsection{Equilibrium prices vs. cash flow jump intensity}

TABLE 3.

The table represents the impacts of the jump intensity $(\lambda)$ with the baseline parameter values.

\begin{tabular}{|l|l|l|l|l|l|}
\hline$\lambda$ & 0.01 & 0.5 & 1 & 1.5 & 2 \\
\hline$\delta_{B}$ & 3.5993 & 3.6027 & 3.6048 & 3.6062 & 3.6077 \\
\hline$\delta_{\bar{B}}$ & 5.9650 & 5.9750 & 5.9787 & 5.9875 & 5.9950 \\
\hline$\delta_{K}$ & 12.6275 & 12.7575 & 12.8850 & 13.0094 & 13.1350 \\
\hline$\varphi^{e}$ & 0.0680 & 0.0949 & 0.1270 & 0.1634 & 0.2039 \\
\hline$\varphi^{o}$ & 0.4001 & 0.5104 & 0.6327 & 0.7671 & 0.9116 \\
\hline$E^{e}\left(\delta_{0}, \varphi^{e}\right)$ & 51.6022 & 48.6310 & 45.5160 & 42.3439 & 39.1383 \\
\hline $\bar{E}^{o}\left(\delta_{0}, \varphi^{o}\right)$ & 43.2846 & 39.0529 & 34.8078 & 30.5786 & 26.4281 \\
\hline
\end{tabular}

TABLE 4.

The table represents the impacts of the jump intensity $(\lambda)$ with $\eta_{1}=$ $8, \eta_{2}=13$ and the baseline parameter values.

\begin{tabular}{|l|l|l|l|l|l|}
\hline$\lambda$ & 0.01 & 0.5 & 1 & 1.5 & 2 \\
\hline$\delta_{B}$ & 3.5985 & 3.5573 & 3.5153 & 3.4733 & 3.4314 \\
\hline$\delta_{\bar{B}}$ & 6.3375 & 6.2950 & 6.2550 & 6.2097 & 6.1675 \\
\hline$\delta_{K}$ & 12.5950 & 12.5975 & 12.6050 & 12.6050 & 12.6094 \\
\hline$\varphi^{e}$ & 0.0675 & 0.0659 & 0.0644 & 0.0629 & 0.0615 \\
\hline$\varphi^{o}$ & 0.4789 & 0.4624 & 0.4473 & 0.4314 & 0.4169 \\
\hline$E^{e}\left(\delta_{0}, \varphi^{e}\right)$ & 51.6720 & 52.1712 & 52.6799 & 53.1879 & 53.6951 \\
\hline $\bar{E}^{o}\left(\delta_{0}, \varphi^{o}\right)$ & 40.6813 & 41.4126 & 42.1201 & 42.8724 & 43.5894 \\
\hline
\end{tabular}

Table 3 and 4 describe the impacts of the jump intensity $\lambda$ of the cash flow on default thresholds, exercise boundary, guarantee costs and equilibrium prices. For an obvious reason, we consider two cases: (i) The mean jump value of the cash flow is negative; (ii) The mean value is positive.

In Table 3, we assume the cash flow has a negative mean jump value $\xi=$ $\frac{p}{\eta_{1}}-\frac{q}{\eta_{2}}=-0.017$. Under this case, as we expected, the default thresholds $\delta_{B}, \delta_{\bar{B}}$, exercise boundary $\delta_{K}$, and guarantee costs $\varphi^{e}$ and $\varphi^{o}$ increase with 
the jump intensity $\lambda$. Naturally, the values $E^{e}\left(\delta_{0}, \varphi^{e}\right)$ and $\bar{E}^{o}\left(\delta_{0}, \varphi^{o}\right)$ of equity held by the entrepreneur decrease with the jump intensity $\lambda$, since the larger the guarantee costs $\varphi^{e}$ and $\varphi^{o}$, the less the amount of equity left to the entrepreneur. Table 3 also says that EGS is better than OGS for the entrepreneur while keeping the other two sides of the agreement the same, and the advantage increases slowly with the jump intensity.

On the contrary to Table 3, we assume the mean jump value is positive in Table 4: $\xi=\frac{p}{\eta_{1}}-\frac{q}{\eta_{2}}=0.004$. Roughly speaking, the opposite holds true as seen in the table.

\section{CONCLUSIONS}

In this paper, we discuss the equilibrium pricing of corporate securities under EGS and OGS respectively. Unlike Yang and Zhang (2013) who consider only EGS in a continuous model, we suppose that the cash flow follows a jump-diffusion process and also study a much more complex swap, OGS.

As pointed out in Kou (2002), the major advantages of the jump-diffusion model are: (i) It can lead to the asymmetric leptokurtic feature and to the implied "volatility smile"; (ii) It has a good analytical tractability.

We present explicit equilibrium prices of all contingent claims involved in the two swaps. As far as OGS is concerned, the default thresholds before and after the option is exercised are somewhat different from each other. The two thresholds determined by a firm that enters into OGS and the exercising boundary of the option determined by its holder (the insurer) constitute a Nash equilibrium, which is explicitly derived in this paper. Based on these conclusions, we provide numerical analysis, which explains what and how factors involved in the two swaps should be taken into account in the agreement. For example, the larger the correlation between the firm and the market, the higher the guarantee costs should be charged and the less the value of equity held by the firm. Such important relationships are, however, generally ignored in reality. Specifically, in sharp contrast to Yang and Zhang (2015), who assume the borrower is risk-averse towards idiosyncratic risk, here EGS is better than OGS as far as the borrower is concerned. The advantage increases dramatically with the cashflow risk.

In our paper, for simplicity, we assume the guaranteed bank loan is a console bond. Theory and practice would predict that a lender or loan insurer would require a short maturity so that the financial condition of the firm could be frequently monitored and financing could be cut off if the firm did poorly. In addition, we do not consider optimal capital structure, the asset substitution effect and debt overhang problem to save the space. However, these issues are also important and so we leave them for future research. 


\section{APPENDIX A}

\section{A PROOF OF THAT THE MEASURE $\mathbb{Q}$ IS INDEPENDENT OF THE UTILITY $U$}

Let $\mathcal{G}_{t} \equiv \sigma\left\{Z_{s}^{1} ; 0 \leq s \leq t\right\}$. It is easy to derive that the optimal consumption $C_{t}^{*}$ is $\mathcal{G}_{t}$ measurable, i.e. $C_{t}^{*} \in \mathcal{G}_{t}$. Hence, we get the stochastic discount factor $\pi_{t} \equiv U_{C_{t}}^{\prime}\left(t, C_{t}^{*}\right) \in \mathcal{G}_{t}$ and the density process $\psi$ for its equivalent martingale measure $\mathbb{Q}$ satisfies $\psi_{t}=\exp (r t) \pi_{t} / \pi_{0} \in \mathcal{G}_{t}$. Utilizing the well known martingale representation theorem, there is $\mathcal{G}$-adapted process $\chi$, such that $d \psi_{t}=\chi_{t} \psi_{t} d Z_{t}^{1}$. Since $\exp (-r t) M_{t}$ must be a martingale under $\mathbb{Q}$, we therefore derive $\chi_{t}=-\eta$ for all $t \geq 0$, following the Girsanov-Meyer theorem. That is, no matter what the utility function in (4) is, we obtain the same measure $\mathbb{Q}$ given by (5). This concludes the proof.

\section{APPENDIX B}

\section{A PROOF OF EQUATIONS (9) and (10)}

First, the boundary condition (10) holds obviously. Second, we get for $\delta_{0} \in \mathcal{D}$ that

$$
\int_{0}^{t} e^{-r s}\left(a_{1} \delta_{s}+a_{2}\right) d s+e^{-r t} V\left(\delta_{t}\right)=\mathbb{E}^{\mathbb{Q}}\left[\int_{0}^{T_{D}} e^{-r s}\left(a_{1} \delta_{s}+a_{2}\right) d s+e^{-r T_{D}} g\left(\delta_{T_{D}}\right) \mid \mathcal{F}_{t}\right] .
$$

Accordingly, the process $\int_{0}^{t} e^{-r s}\left(a_{1} \delta_{s}+a_{2}\right) d s+e^{-r t} V\left(\delta_{t}\right)$ is a martingale if we stop the process once $\delta$ hits the boundary $\partial \mathcal{D}$ and consequently, the drift of its differential is zero. For this reason, by utilizing Ito-Doeblin formula for one jump process, it is not difficult to derive (9).

\section{REFERENCES}

Dao, T. B., 2003. A structure model with jump-diffusion processes. Association Française de Finance.

Duffie, D., 2001. Dymaic asset pricing theory, 3rd ed. Princeton University Press.

Fidrmuc, J., P. Ciaian, and J. Pokrivcak, 2013. Credit constraints, heterogeneous firms and loan defaults. Annals of Economics and Finance 14(1), 53-68.

Goetzmann, W. N., J. E. Ingersoll, and S. A. Ross, 2003. High-water marks and hedge fund management contracts. Journal of Finance 58(4), 1685-1718.

Goldstein, R., N. Ju, and H. Leland, 2001. An EBIT-based model of dynamic capital structure. Journal of Business 74(4), 483-511.

Ingersoll, J.E., 2006. The subjective and objective evaluation of incentive stock options. Journal of Business 79(2), 453-487.

Kou, S. G., 2002. A jump diffusion model for option pricing. Mansgement Science 48(8), 1086-101. 
Kou, S. G. and H. Wang, 2003. First passage times for a jump diffusion process. Advanced in Applied Probability 35, 504-531.

Kou, S. G. and H. Wang, 2004. Option pricing under a double exponential jump diffusion model. Management Science 50(9), 1178-1192.

Leland, H. E., 1994. Corporate debt value, bond covenants, and optimal capital structure. Journal of finance 49, 1213-1252.

Merton, R. C., 1976. Option pricing when underlying stock returns are discontinuous. Journal of Financial Economics 3(1-2), 125-144.

Protter, P., 1990. Stochastic integration and differential equations - a new approach. Springer-Verlag.

Rogers, L. C. G., 2013. Optimal investment. Springer-Verlag.

Sepp, A., 2004. Analytical pricing of double-barrier options under a doubleexponential jump diffusion process: applications of laplace transform. International Journal of Theoretical and Applied Finance 7(2), 151-175.

Suzuki, A. and K. Sawaki, 2009. Double exponential jump diffusion processes and its application to real options. The English International Symposium on Operations Research and Its Applications, Zhangjiajie, China, September 20-22.

Wang, H., Z. Yang, and H. Zhang, 2015. Entrepreneurial finance with equity-forguarantee swap and idiosyncratic risk. European Journal of Operational Research 241(3), 863-871.

Yang, Z. and H. Zhang, 2013. Optimal capital structure with an equity-for-guarantee swap. Economics Letters 118, 355-359.

Yang, Z. and C. Zhang, 2015. Two new equity default swaps with idiosyncratic risk. International Review of Economics and Finance 37, 254-273 